\title{
Biological Control of Root-Knot Nematodes by Organic Acid-Producing Lactobacillus brevis WiKim0069 Isolated from Kimchi
}

\author{
Hye Jeong Seo ${ }^{1 \dagger}$, Ae Ran Park ${ }^{1 \dagger}$, Seulbi Kim ${ }^{1}$, Jehyeong Yeon ${ }^{1}$, Nan Hee Yu ${ }^{1}$, Sanghyun Ha ${ }^{2}$, Ji Yoon Chang ${ }^{2}$, \\ Hae Woong Park (D) ${ }^{2 *}$, and Jin-Cheol Kim (D) ${ }^{1 *}$ \\ ${ }^{l}$ Department of Agricultural Chemistry, Institute of Environmentally Friendly Agriculture, College of Agriculture and \\ Life Science, Chonnam National University, Gwangju 61186, Korea \\ ${ }^{2} R \& D$ Division, World Institute of Kimchi, Gwangju 61755, Korea
}

(Received on August 27, 2019; Revised on September 26, 2019; Accepted on October 31, 2019)

Root-knot nematodes (RKNs) are among the most destructive plant-parasites worldwide, and RKN control has been attempted mainly using chemical nematicides. However, these chemical nematicides have negative effects on humans and the environment, thus necessitating the search for eco-friendly alternative RKN control methods. Here, we screened nematicidal lactic acid bacteria (LAB) isolated from kimchi and evaluated their efficacy as biocontrol agents against RKNs. Of 237 bacterial strains, Lactobacillus brevis WiKim0069 showed the strongest nematicidal activity against the second-stage juveniles (J2) of Meloidogyne incognita, $M$. arenaria, and $M$. hapla and inhibited the egg hatch of $M$. incognita. The culture filtrate of WiKim0069 had a pH of 4.2 and contained acetic acid $(11,190 \mu \mathrm{g} / \mathrm{ml})$, lactic acid $(7,790 \mu \mathrm{g} / \mathrm{ml})$, malic acid $(470 \mu \mathrm{g} / \mathrm{ml})$, and succinic acid $(660 \mu \mathrm{g} / \mathrm{ml})$. An artificial mixture of the four or-

\footnotetext{
These authors contributed equally to this work.

*Corresponding author.

Jin-Cheol Kim

Phone) +82-62-530-2132, FAX) +82-62-530-2139

E-mail)kjinc@jnu.ac.kr

Hae Woong Park

Phone) +82-62-610-1728, FAX) +82-62-610-1810

E-mail) haewoong@wikim.re.kr

ORCID

Jin-Cheol Kim

https://orcid.org/0000-0003-0753-1966

Hae Woong Park

https://orcid.org/0000-0002-5181-1255

(c) This is an Open Access article distributed under the terms of the Creative Commons Attribution Non-Commercial License (http:// creativecommons.org/licenses/by-nc/4.0) which permits unrestricted noncommercial use, distribution, and reproduction in any medium, provided the original work is properly cited.
}

Articles can be freely viewed online at www.ppjonline.org. ganic acids produced by WiKim0069 also induced $98 \%$ M. incognita $\mathrm{J} 2$ mortality at a concentration of $1.25 \%$, indicating that its nematicidal activity was derived mainly from the four organic acids. Application of WiKim0069 culture filtrate suppressed the formation of galls and egg masses on tomato roots by M. incognita in a dose-dependent manner in a pot experiment. The fermentation broth of WiKim0069 also reduced gall formation on melon under field conditions, with a higher efficacy $(62.8 \%)$ than that of fosthiazate $(32.8 \%)$. This study is the first report to identify the effectiveness of kimchi LAB against RKNs and to demonstrate that the organic acids produced by LAB can be used for the RKN management.

Keywords : lactic acid bacteria, Lactobacillus brevis, Meloidogyne spp., nematicidal activity, organic acid

Handling Editor : Lee, Yong Hoon

Root-knot nematodes (RKNs; Meloidogyne spp.), among the most destructive agricultural pests, are responsible for a large portion of the considerable economic losses, estimated at about $\$ 77$ billion a year caused by phytopathogenic nematodes worldwide (Ralmi et al., 2016; Yadav, 2017). Of the more than 100 Meloidogyne species, M. incognita, $M$. hapla, M. javanica, and M. arenaria are considered the most widespread and economically important plantparasitic RKNs (Elling, 2013; Jones et al., 2013; Molinari, 2009). RKNs are obligate parasites capable of penetrating the roots of host plants belowground, resulting in damaged root tissues that provide infection sites for soil-borne phytopathogenic microorganisms (Molinari et al., 2014); this parasitism results in the poor growth of many horticultural 
and field crops and consequently severe reductions in yield (Blok et al., 2008; Moens et al., 2009).

Soil-borne diseases caused by RKNs can spread easily and are very difficult to control because of their high reproduction rate and short generation time (Trudgill and Blok, 2001). The extensive use of synthetic pesticides has long been a management strategy for the control of RKNs. However, the increasing public concern about the negative effects of the continuous usage of chemical pesticides, especially on human health and the environment, has limited the use of many synthetic chemicals (Aktar et al., 2009). Therefore, there is an urgent need to identify effective and eco-friendly alternatives based on microorganisms to reduce the use of chemical nematicides in agriculture.

Lactic acid bacteria (LAB) have traditionally been used to ferment carbohydrate-rich foods. $\mathrm{LAB}$ are known to improve the nutritional value of foods and control human intestinal infections (Gilliland, 1990; Lindgren and Dobrogosz, 1990). LAB are generally recognized as safe by the United States Food and Drug Administration (FDA) because of their ubiquitous appearance in food and their probiotic potential on human mucosal surfaces (Stiles and Holzapfel, 1997). They also have an antagonistic activity against pathogenic bacteria and fungi, which makes them ideal for developing biocontrol agents for use on plants (Sitton and Patterson, 1992; Trias et al., 2008). For these reasons, $\mathrm{LAB}$ are currently attracting much attention in the agricultural industry as alternatives to chemical pesticides, which are associated with problems such as antibiotic resistance and pesticide residue.

Kimchi, a traditional fermented food in Korea, is plantbased and associated with a number of LAB such as Lactobacillus and Leuconostoc species, including L. plantarum, L. brevis, L. curvatus, L. mesenteroides, and L. citreum (Jung et al., 2011). Many studies have demonstrated that Lactobacillus species exert an inhibitory effect on pathogenic bacteria in human intestinal epithelial cells (Guarner et al., 2012; Jang et al., 2011; Lim et al., 2018). Specifically, several compounds produced by $\mathrm{LAB}$, such as organic acids, hydrogen peroxide, bacteriocins, and fat and amino acid metabolites, are among the antimicrobial factors responsible for the competitive exclusion of pathogenic bacteria (Barefoot and Klaenhammer, 1983; Kormin et al., 2001). L. plantarum of Lactobacillus species has been known to function as an antagonistic agent and exhibit antimicrobial activity (Hamed et al., 2011), but little is known about the potential of $L$. brevis as a biocontrol agent against phytopathogens. Additionally, there are no reports on the nematicidal activity of LAB $L$. brevis on phytopathogenic nematodes.
In the course of screening of nematicidal LAB strains, we found that $L$. brevis WiKim0069 isolated from kimchi showed strong nematicidal activity against $M$. incognita. Therefore, the objectives of this study were (1) to examine the in vitro nematicidal activity of the culture filtrate of WiKim0069 strain against three Meloidogyne species, (2) to identify and characterize the nematicidal metabolites produced by WiKim0069, and (3) to evaluate the efficacy of WiKim0069 as a biocontrol agent in controlling RKNs on vegetable and fruit plants.

\section{Materials and Methods}

Isolation of $\mathrm{LAB}$ from kimchi. $\mathrm{LAB}$ were isolated from kimchi prepared in homes and temples across the Republic of Korea. Kimchi samples (500 g) were ground using a hand blender for $2 \mathrm{~min}$. The obtained kimchi juice was filtered through a sterilized cheesecloth, serially diluted with saline solution $(0.85 \%$; $3 \mathrm{M}$, St. Paul, MN, USA), and spread onto de Man-Rogosa-Sharpe (MRS) agar (Oxoid, Basingstoke, New Hampshire, England) plates containing $\mathrm{CaCO}_{3}\left(2 \%\right.$, w/v). The plates were incubated $30^{\circ} \mathrm{C}$ under anaerobic conditions for 2 days, and the tentatively considered LAB strains were selected.

Nematode inoculum. The root-knot nematodes $M$. incognita, M. hapla, and $M$. arenaria were acquired from the Korea Research Institute of Chemical Technology (Republic of Korea), Seoul National University (Republic of Korea), and National Institute of Agricultural Science and Technology (Republic of Korea), respectively (Hwang et al., 2014; Kim et al., 2001; Park et al., 2014).

Three Meloidogyne species were maintained on tomato (Solanum lycopersicum Mill. cv. Seokwang) in a greenhouse at $25^{\circ} \mathrm{C}$ with day/night lighting of $16 \mathrm{~h} / 8 \mathrm{~h}$. The eggs were extracted in $1 \%$ sodium hypochlorite solution from the infected tomato plants and the egg suspension was immediately rinsed twice with sterile water (Yeon et al., 2019). The collected eggs was used for in vitro and pot experiments. The $\mathrm{J} 2$ that hatched after 5 days of incubation at $28^{\circ} \mathrm{C}$ were collected using the Baermann funnel method (Viglierchio and Schmitt, 1983) and used to evaluate the nematicidal effects of LAB.

Screening of nematicidal $\mathrm{LAB}$. The isolated $\mathrm{LAB}$ were added to MRS broth (Becton, Dickinson and Company, Franklin Lakes, NJ, USA) and grown anaerobically without shaking at $30^{\circ} \mathrm{C}$. One-day cultured $\operatorname{LAB}(0.1 \%, v / v)$ were inoculated into fresh MRS broth $(50 \mathrm{ml})$ and incubated at $30^{\circ} \mathrm{C}$ under anaerobic conditions for $24 \mathrm{~h}$. The LAB culture 
was centrifuged at $3,000 \mathrm{rpm}$ for $10 \mathrm{~min}$ at $4^{\circ} \mathrm{C}$, and the $\mathrm{pH}$ of supernatant was measured using a calibrated digital $\mathrm{pH}$ meter (NeoMet pH-200L, Seoul, Korea). The supernatant was then filtered $(0.20 \mu \mathrm{m})$. The culture filtrates of LAB were stored at $-20^{\circ} \mathrm{C}$ until use.

The nematicidal activity of LAB was evaluated using their effect on the mortality of $M$. incognita $\mathrm{J} 2$. Treatments were performed in 96 well tissue culture plates using approximately $50 \mathrm{~J} 2 /$ replicate (Ashoub and Amara, 2010; Meyer et al., 2000; Terefe et al., 2009). To prevent solution evaporation, the plates were covered and kept in the dark at $25^{\circ} \mathrm{C}$, with gentle shaking. Three days after exposure, the J2 were observed under a light microscope (Leica DM IL LED, Leica Microsystems CMS GmbH, Wetzlar, Germany). Three days after exposure, the $\mathrm{J} 2$ were moved to plain water and grouped into motile and immotile categories based on observations made after pricking their bodies with a fine needle. Nematodes that did not move and retained a stiff and straight body shape even after pricking with a needle were considered dead (Cayrol et al., 1989). Sterilized distilled water was used as a negative control. The experiment was repeated twice with three replicates. To analyze the nematicidal activity of LAB against $M$. incognita, J2 mortality was converted to percentage mortality and corrected using the formula of Schneider-Orelli (Schneider and Orelli, 1947): Mortality $(\%)=[($ mortality percentage in treatment - mortality percentage in the negative control)/ $(100-$ mortality percentage in the negative control) $] \times 100$. The nematicidal LAB were first screened based on the induction of more than $90 \%$ mortality at a culture filtrate concentration of $2.5 \%$ against the $\mathrm{J} 2$ of $M$. incognita. The nematicidal activities of the screened LAB were evaluated again using $M$. incognita $\mathrm{J} 2$ mortality over a concentration range of $0.63 \%$ to $2.5 \%$.

Molecular identification of WiKim0069. A WiKim0069 isolate showing nematicidal activity was identified by $16 \mathrm{~S}$ rRNA nucleotide sequence analysis. The genomic DNA of the WiKim0069 isolate was prepared using a DNeasy Blood and Tissue kit (Qiagen, Hilden, Germany) following the manufacturer's recommendations. PCR amplification of the 16S rRNA gene was performed using the universal bacterial primer pair 9F (5'-GAGTTTGATCCTGGCTCAG3')/1512R (5'-ACGGCTACCTTGTTACGACTT-3') in a $20-\mu \mathrm{l}$ reaction mixture containing genomic DNA $(2 \mu \mathrm{l})$, primers $(1 \mu \mathrm{l}$ of each, $10 \mathrm{pM})$, sterilized distilled water (16 $\mu 1)$, and Accupower PCR premix $(1 \mu \mathrm{l})$ (Bioneer Corp., Daejeon, Korea). The species was identified by comparison with the GenBank reference sequence database using BLASTn. Sequence alignment and phylogenetic analysis were performed using MEGA 6 with the neighbor-joining (NJ) method, with the number of bootstrap trials set to 1,000. The Kimura 2-parameter model was selected as the best model to construct the tree for NJ (Tamura et al., 2013).

In vitro nematicidal activity of WiKim0069 culture filtrate. The nematicidal activity of the culture filtrate of WiKim0069 was evaluated by assessing its effect on $M$. incognita, $M$. arenaria, and M. hapla $\mathrm{J} 2$ mortality and hatching inhibition of $M$. incognita eggs over a concentration range of $0.63 \%$ to $2.5 \%$. Approximately $50 \mathrm{~J} 2$ and 150 eggs per well were used for the bioassay in 96 well tissue culture plates. Sterilized distilled water was used as the negative control. The $\mathrm{J} 2$ mortality of three Meloidogyne species was determined in the same method as described in Section Screening of nematicidal LAB. The ability of LAB to inhibit $M$. incognita $\mathrm{J} 2$ hatching was determined at 7 days after exposure according to Abbott's formula (Abbott, 1925): J2 hatching inhibition (\%) $=[(\mathrm{J} 2$ percentage in the negative control $-\mathrm{J} 2$ percentage in treatment $/ \mathrm{J} 2$ percentage in the negative control] $\times 100$. All experiments were conducted with three replicates and repeated twice.

Analysis of organic acids produced by WiKim0069. The concentrations of organic acids (acetic, citric, fumaric, lactic, malic, malonic, oxalic, succinic, and tartaric acids) in the culture filtrate of WiKim0069 were determined. For organic acid analysis, WiKim0069 was cultured in a flask $(500 \mathrm{ml})$ containing MRS broth $(100 \mathrm{ml})$ under anaerobic conditions at $30^{\circ} \mathrm{C}$ for $24 \mathrm{~h}$, and a seed culture $(1 \%, \mathrm{v} / \mathrm{v})$ was inoculated in liquid medium (3 1) containing maltose $(4 \%)$, yeast extract $(2 \%)$, sodium acetate $(0.5 \%)$, dipotassium phosphate $(0.2 \%)$, magnesium sulfate $(0.01 \%)$, and manganese sulfate $(0.005 \%)$ in a jar bioreactor $(51$, MARADO-05D-XS, BioCnS, Daejeon, Korea). The cultivation was conducted at $30^{\circ} \mathrm{C}$ for $24 \mathrm{~h}$ with an agitation speed of $300 \mathrm{rpm}$ and an aeration rate of $1.0 \mathrm{vvm}$ (volume of air added to the liquid volume per minute). The culture broth was centrifuged at $5,000 \mathrm{rpm}$ for $10 \mathrm{~min}$ at $4^{\circ} \mathrm{C}$, and the supernatant was filtered $(0.45 \mu \mathrm{m})$ and injected into a chromatograph with a loop of $50 \mu \mathrm{l}$ using an autosampler with a fixed volume of $10 \mu \mathrm{l}$.

High-performance liquid chromatography (HPLC; Waters Alliance e2695 system, Milford, MA, USA) was used for the analysis of organic acids. Chromatographic separation was carried out isocratically at $50^{\circ} \mathrm{C}$ with a mobile phase of $0.008 \mathrm{~N}$ sulfuric acid at a flow rate of $0.6 \mathrm{ml} / \mathrm{min}$ on an Aminex HPX-87H reverse-phase column $(300 \times 7.8$ mm, $9 \mu \mathrm{m}$ particle size; Bio-Rad, Hercules, CA, USA). 
HPLC equipment consisted of a quaternary pump, an online degasser, a column oven, and a UV-visible detector (2489, Waters). The UV detector was set at $210 \mathrm{~nm}$ for the detection of organic acids. Data were collected and processed on a computer with EMPOWER 3 software (Waters). Quantification was performed using the peak areas from the external calibration with standard solutions.

In vitro nematicidal activity of organic acids. Based on the HPLC analysis of organic acids, an artificial mixture of organic acids was prepared with the same composition as the mixture produced by WiKim0069. The mixture of organic acids contained acetic acid $(11,190 \mu \mathrm{g} / \mathrm{ml})$, lactic acid $(7,790 \mu \mathrm{g} / \mathrm{ml})$, malic acid $(470 \mu \mathrm{g} / \mathrm{ml})$, and succinic acid $(660 \mu \mathrm{g} / \mathrm{ml})$ in a liquid medium. The effect of the organic acid mixture on J2 mortality of $M$. incognita was evaluated over the range of $0.63 \%$ to $10 \%$. Sterilized distilled water and the culture filtrate of WiKim0069 were used as the negative control and the positive control, respectively. The effects of the four organic acids produced by WiKim0069 on $\mathrm{J} 2$ mortality of $M$. incognita were assessed in two-fold serial dilutions at concentrations of $0-10,000 \mu \mathrm{g} / \mathrm{ml}$. All experiments were repeated twice with three replicates.

\section{Efficacy of WiKim0069 for biocontrol of M. incognita} on tomato plants in pot experiments. Pot experiments were carried out to evaluate the efficacy of WiKim0069 against RKN infection on tomato. Seokwang tomato seeds were sown in horticultural nursery soil at $25^{\circ} \mathrm{C}$ for 4 weeks. Tomato seedlings at the five-leaf stage were transplanted in $9.5 \mathrm{~cm}$ diameter pots containing a sterilized nursery soil-sand mix $(1: 1, \mathrm{v} / \mathrm{v})$ and inoculated with $M$. incognita (10,000 eggs); this was followed by treatment with WiKim0069 culture filtrates $(20 \mathrm{ml}, 5$ - and 20-fold dilutions) by soil drench. Each treatments were compared with Tween 20-treated tomato plants $(20 \mathrm{ml}, 250 \mu \mathrm{g} / \mathrm{ml}$, Sigma-Aldrich Co., St Louis, MO, USA), which were used as the untreated negative control. Commercial nematicide Sunchungtan containing $30 \%$ fosthiazate $(20 \mathrm{ml}$, SL, 4,000fold dilution; Farm Hannong Co., Seoul, Korea) was used as the positive control. Six weeks after inoculation, the plants were uprooted, washed to remove the soil, and rated for galls. Galling of root systems was rated using a 0-5 severity scale (Barker, 1985). The galling index (GI) was assigned as follows: $0=0-10 \%, 1=11-20 \%, 2=21-50 \%$, $3=51-80 \%, 4=81-90 \%$, and $5=91-100 \%$ root galls. The control value was determined using the following equation (Seo and Kim, 2014): Control value (\%) = [(GI of untreated negative control - GI of treatment)/GI of untreated negative control] $\times 100$. The number of egg masses was counted after staining in phloxine B solution (Holbrook et al., 1983). Galled roots were placed in an aqueous solution of phloxine $B(0.15 \mathrm{mg} / \mathrm{ml}$ tap water) for $15 \mathrm{~min}$ and rinsed with tap water to remove the residual stain on the roots. The number of egg masses was converted to the control value using the following formula: Control value $(\%)=$ [(the number of egg masses of untreated negative control the number of egg masses of treatment)/the number of egg masses of untreated negative control] $\times 100$. Each treatment was replicated twice, and each replicate consisted of four tomato plants. The experiment was repeated twice.

Efficacy of WiKim0069 for biocontrol of Meloidogyne spp. on melon plants under field conditions. The field experiment was performed in June-August 2017 in a melon greenhouse naturally infested with Meloidogyne spp., located in Jawang-li, Buyeo-eub, Buyeo, Chungnam Province, Republic of Korea. The initial population density of Meloidogyne spp. was 1,105 $\pm 287 / 100 \mathrm{~g}$ of soil. Melon (Cucumis melo cv. Earls Favorite) seeds were sown in seed trays containing nursery soil in a greenhouse and then transplanted into the plots after 3 weeks. The plants were arranged according to a randomized complete block design. Each treatment consisted of three replicates, with 15 melon plants in a $4 \mathrm{~m}^{2}\left(1 \times 4 \mathrm{~m}^{2}\right)$ plot/replicate. The treatments were (1) untreated control, (2) 5-fold dilution of the fermentation culture of L. brevis WiKim0069, and (3) 4000-fold dilution of fosthiazate (30\% SL, Sunchungtan). Based on the results of the pot experiments, L. brevis WiKim0069, which had the best efficacy against RKNs (5fold diluted culture filtrates), was used in the field trials. Tween $20(250 \mu \mathrm{g} / \mathrm{ml}$, Sigma-Aldrich Co. $)$ was used as the untreated control. Fosthiazate (30\% SL, Sunchungtan) was used as the positive control. Four days after transplanting, each treatment $(100 \mathrm{ml})$ was applied four times to each plant by soil drench at 10 -day intervals. Sixty days after the first treatment, the plants were harvested, and the root systems were rated for galls. Additionally, 30 and 60 days after the first treatment, soil samples $(200 \mathrm{~g})$ were taken from around the melon roots at a depth of $25-30 \mathrm{~cm}$ using a $30 \mathrm{~cm}$ diameter auger (three samples/replicate) and transported to the laboratory in polythene bags for further processing. The RKN population density in the soil was determined using the Baermann funnel method (Hwang et al., 2014; Jenkins, 1964).

Statistical analysis. The parameters measured in this study were designed to evaluate the nematicidal activity and efficacy of organic acids and WiKim0069 against RKNs. Analyses were conducted separately for the in vitro and the 


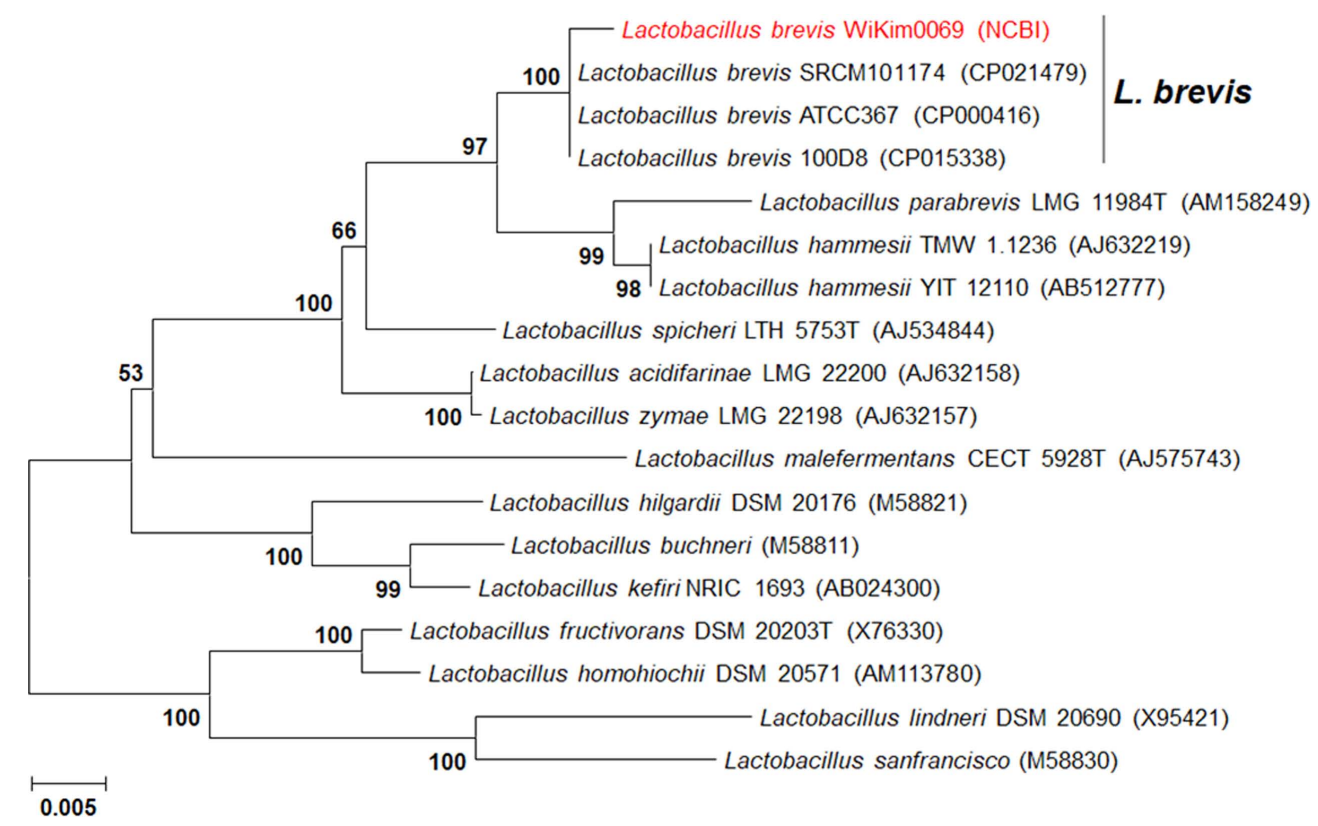

Fig. 1. Phylogenetic trees derived from the distance analysis of the $16 \mathrm{~S}$ rRNA gene sequences of WiKim0069. Sequences were aligned using MEGA 6.0 software. Phylogenetic trees were constructed using the neighbor-joining method with bootstrap analysis (1,000 trials). Bars indicate the percentage of sequence divergence.

pot and field experiments. All data were analyzed for the homogeneity of variance using SPSS statistical analysis software version 21.0 for Windows (IBM Corp., Armonk, NY, USA). Data are expressed as the mean \pm standard deviation of replicates, and they were evaluated by oneway analysis of variance (ANOVA). Statistical differences among the treatments were determined according to Duncan's multiple-range test $(P<0.05)$.

\section{Results}

Screening and identification of nematicidal LAB. A total of 237 LAB strains were isolated from kimchi collected in the Republic of Korea. Among these, 16 strains exhibited distinct nematicidal activity against $M$. incognita $\mathrm{J} 2$ with the induction of more than $90 \%$ mortality at a culture filtrate concentration of $2.5 \%$ (Supplementary Table 1) and the $\mathrm{pH}$ of their culture was between 3.6 and 4.4 (Supplementary Table 2). Among these strains, the culture filtrate of LAB strain 291 showed the strongest nematicidal activity against $M$. incognita $\mathrm{J} 2$ at day 3 of exposure, with $98.03 \%$ mortality at a culture filtrate concentration of $1.25 \%$ (Supplementary Table 2). The selected LAB strain 291 was designated as WiKim0069 and identified as L. brevis based on BLASTn and phylogenetic analyses of the amplified 16S rRNA gene sequence (Fig. 1). The amplified genes were registered in GenBank under the accession number MH842143. L. brevis WiKim0069 was deposited in the
KCCM (Korean Culture Center of Microorganisms, Seoul, Korea) as KCCM12134P.

In vitro nematicidal activity of $L$. brevis WiKim 0069 . The effects of WiKim0069 culture filtrate on M. incognita J2 mortality and hatching inhibition were determined at concentrations of $0.63 \%$ to $20 \%$. The culture filtrate of WiKim0069 exhibited strong nematicidal activity against $M$. incognita $\mathrm{J} 2$ at day 3 of exposure, with $98.0 \%$ and $100.0 \%$

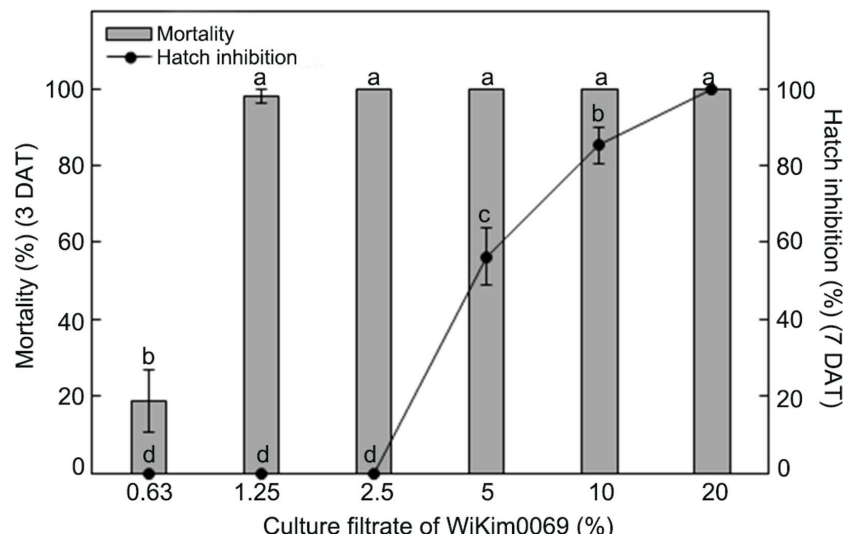

Fig. 2. Effect of the culture filtrate of Lactobacillus brevis WiKim0069 on the mortality of second-stage juveniles and inhibition of the hatching of Meloidogyne incognita. Values are the mean $\pm \mathrm{SD}$ of two runs with three replicates. Means with the same letter are not significantly different $(P<0.05)$ according to Duncan's multiple range test. DAT, days after treatment. 


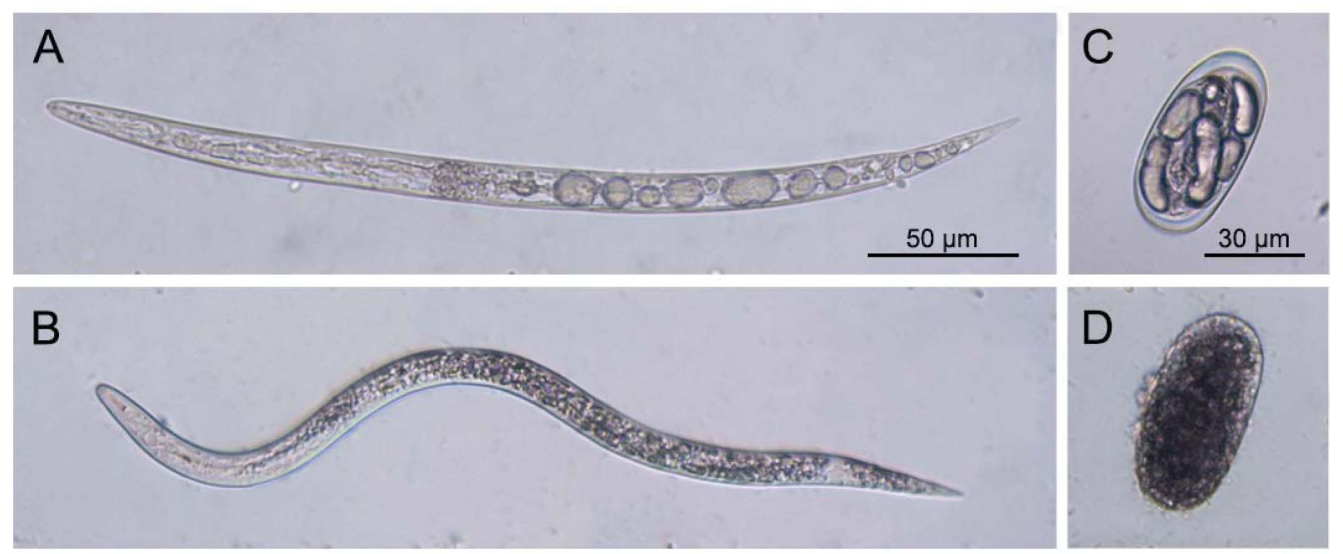

Fig. 3. Effects of Lactobacillus brevis WiKim0069 on second-stage juveniles (J2s) and eggs of Meloidogyne incognita. J2s were treated with the culture filtrate of $2.5 \%$ L. brevis WiKim0069 (A, C) and sterilized distilled water (B, D).

mortality at concentrations of $1.25 \%$ and $2.5 \%$, respectively (Fig. 2). WiKim0069 also induced significant inhibition of $M$. incognita hatching in a dose-dependent manner. Treatment with $5 \%, 10 \%$, and $20 \%$ of the WiKim0069 culture filtrate resulted in $56.2 \%, 85.2 \%$, and $100.0 \%$ inhibition, respectively, of $M$. incognita hatching after 7 days of exposure (Fig. 2).

After treatment with $2.5 \%$ WiKim0069 culture filtrate, all $M$. incognita $\mathrm{J} 2 \mathrm{~s}$ were observed to be dead with rigid and straight bodies, whereas the $\mathrm{J} 2 \mathrm{~s}$ in the untreated control were vigorous and still moving (Fig. 3). The intestinal tract of nematodes treated with $2.5 \% \mathrm{WiKim} 0069$ culture filtrate exhibited pathological changes, as some unusual patch-like structures were clearly visible, which were possibly newly created vacuoles following the destruction of internal or-

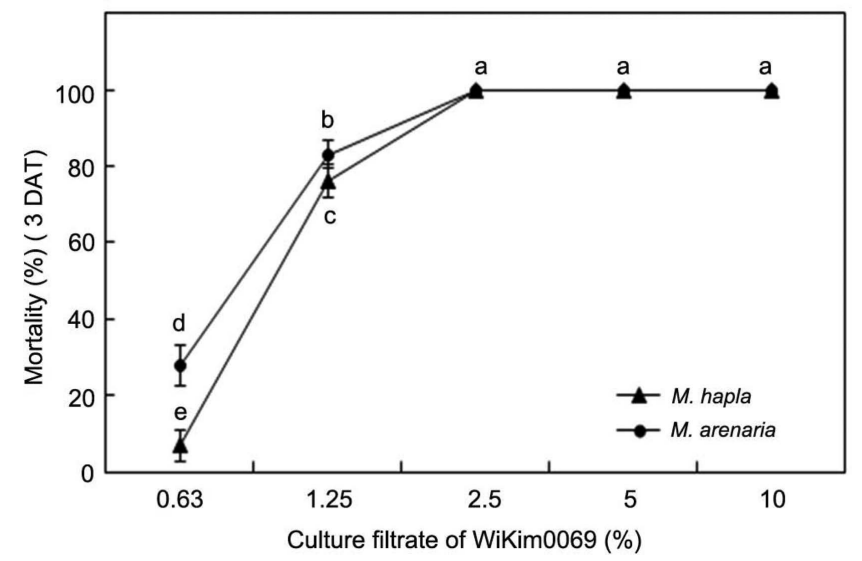

Fig. 4. Effect of the culture filtrate of Lactobacillus brevis WiKim0069 on the mortality of second-stage juveniles of Meloidogyne hapla and Meloidogyne arenaria. Values are the mean \pm SD of two runs with three replicates. Means with the same letter are not significantly different $(P<0.05)$ according to Duncan's multiple range test. DAT, days after treatment. gans by the nematicidal activity of WiKim0069. Additionally, WiKim0069 showed toxicity against $M$. incognita eggs and impaired the normal embryonic development of the juveniles by producing extensive vacuoles inside eggs.

WiKim0069 also showed strong nematicidal activity against other RKN species, $M$. hapla and $M$. arenaria. J2 mortality after $3 \mathrm{~d}$ of exposure at $0.63 \%, 1.25 \%$, and $2.5 \%$ of WiKim0069 culture filtrate was 6.8\%, 76.2\%, and 100\% against $M$. hapla and $27.8 \%, 83.1 \%$, and $100 \%$ against $M$. arenaria, respectively (Fig. 4). Thus, based on the J2 mortality, WiKim0069 exhibited similar nematicidal effects against the major three RKN species $M$. incognita, M. hap$l a$, and $M$. arenaria at concentrations of $1.25 \%$ and $2.5 \%$. However, at a concentration of $0.63 \%$, the nematicidal effect of WiKim0069 against M. incognita was somewhat higher than that against the other two RKN species.

Analysis of organic acids produced by $L$. brevis WiKim0069. HPLC analysis was used to identify the organic acids produced by WiKim0069 strain cultured in liquid

Table 1. Organic acids produced by Lactobacillus brevis WiKim0069 in Lactobacilli MRS broth

\begin{tabular}{lc}
\hline Organic acid & Production $(\mu \mathrm{g} / \mathrm{ml})$ \\
\hline Acetic acid & 11,190 \\
Citric acid & ND \\
Fumaric acid & ND \\
Lactic acid & 7,790 \\
Malic acid & 470 \\
Malonic acid & ND \\
Oxalic acid & ND \\
Succinic acid & 660 \\
Tartaric acid & ND
\end{tabular}

ND, not detected. 
Table 2. Effect of the culture filtrate of Lactobacillus brevis WiKim0069 and the artificial mixture of organic acids on Meloidogyne incognita $\mathrm{J} 2$ mortality

\begin{tabular}{ccc}
\hline $\begin{array}{c}\text { Concentration } \\
(\%)\end{array}$ & $\begin{array}{c}\text { L. brevis } \\
\text { WiKim0069 }\end{array}$ & $\begin{array}{c}\text { Mixture of } \\
\text { organic acids }\end{array}$ \\
\hline 0.63 & $7.86 \pm 8.94 \mathrm{~b}$ & $11.74 \pm 6.78 \mathrm{~b}$ \\
1.25 & $98.03 \pm 1.75 \mathrm{a}$ & $98.05 \pm 2.76 \mathrm{a}$ \\
2.50 & $100 \mathrm{a}$ & $100 \mathrm{a}$ \\
5.00 & $100 \mathrm{a}$ & $100 \mathrm{a}$ \\
\hline
\end{tabular}

Values are the mean \pm SD of two runs with three replicates. Means with the same letter are not significantly different $(P<0.05)$, according to Duncan's multiple range test.

broth. After 1 day of incubation in a jar bioreactor, the fermented culture of WiKim0069 had a pH of 4.2 (Supplementary Table 2 ) and contained $2 \%$ acids, which consisted of acetic acid $(11,190 \mu \mathrm{g} / \mathrm{ml})$, lactic acid $(7,790 \mu \mathrm{g} / \mathrm{ml})$, malic acid $(470 \mu \mathrm{g} / \mathrm{ml})$, and succinic acid $(660 \mu \mathrm{g} / \mathrm{ml})$ (Table 1).

In vitro nematicidal activity of artificial mixture of organic acids. In order to examine the potential of organic acids as nematicidal metabolites, the effects on M. incognita J2 mortality was compared between the culture filtrate of WiKim0069 and the artificial mixture containing the same amounts of organic acids produced by WiKim0069. After 3 days of exposure, the culture filtrate of WiKim0069 induced dose-dependent mortality, and the artificial mixture of organic acids also showed a statistically similar activity

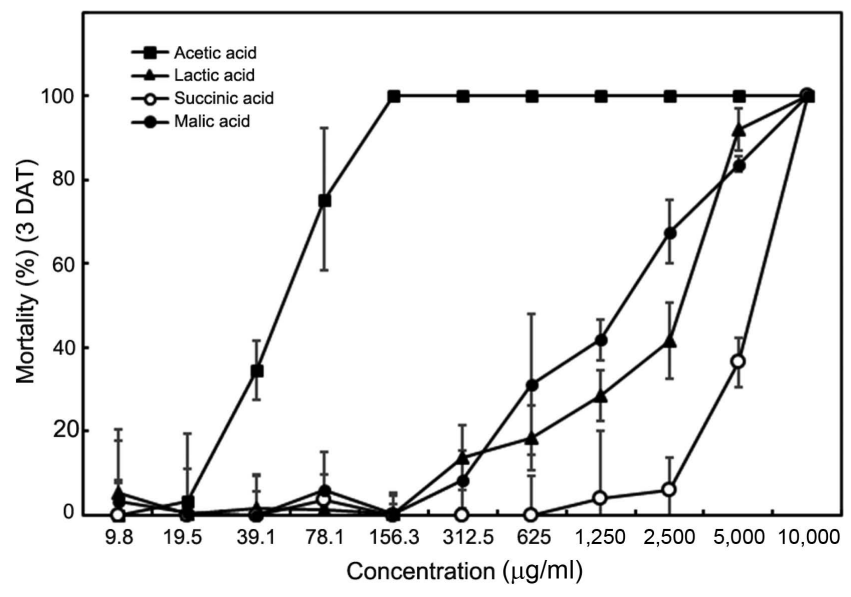

Fig. 5. Effect of acetic acid, lactic acid, malic acid, and succinic acid on the mortality of second-stage juveniles of Meloidogyne incognita. Values are the mean \pm SD of two runs with three replicates. Means with the same letter are not significantly different $(P<0.05)$ according to Duncan's multiple range test. DAT, days after treatment.

against $M$. incognita (Table 2). Furthermore, at concentrations of $1.25 \%$ to $5 \%$, both the WiKim0069 culture filtrate and the artificial mixture of organic acids exhibited same effects against the $M$. incognita J2, suggesting that the nematicidal activity of the culture filtrate of WiKim0069 came from the organic acids.

When the four organic acids produced by WiKim0069 were treated individually with $M$. incognita $\mathrm{J} 2$ at higher concentration $(10,000 \mu \mathrm{g} / \mathrm{ml})$, they completely killed $\mathrm{J} 2$ at

Table 3. Effect of Lactobacillus brevis WiKim0069 culture filtrate on gall index and egg mass on tomato plants infected by Meloidogyne incognita

\begin{tabular}{lccccc}
\hline \multicolumn{1}{c}{ Treatment } & Fold & Gall index (GI) & Control value (GI, \%) & Egg mass (EM) & Control value (EM, \%) \\
\hline WiKim0069 & $\times 5$ & $1.0 \pm 0.0 \mathrm{c}$ & $57.9 \pm 0.0 \mathrm{~b}$ & $36.8 \pm 15.1 \mathrm{~b}$ & $78.9 \pm 8.7 \mathrm{~b}$ \\
WiKim0069 & $\times 20$ & $1.8 \pm 0.3 \mathrm{~b}$ & $26.3 \pm 12.2 \mathrm{c}$ & $46.0 \pm 97.0 \mathrm{~b}$ & $73.6 \pm 21.2 \mathrm{~b}$ \\
Fosthiazate & $\times 4,000$ & $0.0 \pm 0.0 \mathrm{~d}$ & $100.0 \pm 0.0 \mathrm{a}$ & $2.5 \pm 1.7 \mathrm{~b}$ & $98.6 \pm 1.0 \mathrm{a}$ \\
Untreated control & - & $2.4 \pm 0.3 \mathrm{a}$ & - & $174.3 \pm 68.3 \mathrm{a}$ & - \\
\hline
\end{tabular}

Values are the mean \pm SD of two runs with two replicates (four tomato plants/replicate). Means with the same letter are not significantly different $(P<0.05)$, according to Duncan's multiple range test.

Table 4. Efficacy of the fermentation culture of Lactobacillus brevis WiKim0069 on melon plants in a field naturally infected by Meloidogyne spp.

\begin{tabular}{|c|c|c|c|c|}
\hline \multirow{2}{*}{ Treatment } & \multicolumn{2}{|c|}{ No. of nematodes in $100 \mathrm{~g}$ of soil } & \multirow{2}{*}{ Galling index } & \multirow{2}{*}{ Control value $(\%)$} \\
\hline & 30 Days & 60 Days & & \\
\hline WiKim0069 (×5) & $408 \pm 84 \mathrm{a}$ & $744 \pm 559 a$ & $0.8 \pm 0.2 \mathrm{~b}$ & $62.8 \pm 6.5 \mathrm{a}$ \\
\hline Fosthiazate $(\times 4,000)$ & $267 \pm 67 \mathrm{a}$ & $886 \pm 47 \mathrm{a}$ & $1.5 \pm 0.6 \mathrm{ab}$ & $32.8 \pm 27.1 \mathrm{~b}$ \\
\hline Untreated control & $729 \pm 510 a$ & $1,189 \pm 910 \mathrm{a}$ & $2.3 \pm 0.4 \mathrm{a}$ & - \\
\hline
\end{tabular}

Values are the mean \pm SD of three replicates ( 15 melon plants/replicate). Each treatment consisted of 45 melon plants. Means with the same letter are not significantly different $(P<0.05)$, according to Duncan's multiple range test. 
day 3 of exposure (Fig. 5). In addition, treatment with 5,000 $\mu \mathrm{g} / \mathrm{ml}$ acetic acid, lactic acid, malic acid, and succinic acid induced $100 \%, 91.96 \%, 83.76 \%$, and $36.44 \%$ mortality at day 3 of exposure, respectively. These results indicated that among the four organic acids analyzed in this study, acetic acid showed the strongest nematicidal activity, with $100 \%$ $\mathrm{J} 2$ mortality at a concentration of $156.3 \mu \mathrm{g} / \mathrm{ml}$.

\section{Efficacy of WiKim0069 for biocontrol of M. incognita} on tomato plants in pot experiments. The treatments with 5-fold and 20-fold dilutions of the culture filtrate of WiKim0069 reduced gall formation by $57.9 \%$ and $26.3 \%$, respectively, compared to the untreated control treatment (Table 3). The inhibitory activity of WiKim0069 on gall and egg mass formation on tomato was weaker than that of fosthiazate. However, WiKim0069 (5-fold dilution) suppressed gall formation on tomato with significantly lower gall index values than those of the untreated control. Additionally, the egg mass formation on tomato treated with the culture filtrate of WiKim0069 was significantly lower than that on the untreated control.

Efficacy of WiKim0069 for biocontrol of Meloidogyne spp. on melon plants under field conditions. The fermentation culture of WiKim0069, at 5-fold dilution, effectively suppressed gall formation on melon by $62.8 \%$ in fields naturally infested with Meloidogyne spp., which was significantly higher than that observed for fosthiazate (32.8\%) (Table 4). The RKN density in the soil (100 g) treated with WiKim0069 was significantly lowered to 408 and 744 nematodes 30 and 60 days after first exposure, respectively, than the RKN density in the untreated soil of 729 and 1,189 nematodes, respectively. Additionally, the RKN density in the soil treated with WiKim0069 (408 nematodes $/ 100 \mathrm{~g}$ ) was 1.5 times higher than that in the soil treated with fosthiazate ( 267 nematodes $/ 100 \mathrm{~g}$ ) 30 days after first exposure. However, 60 days after first exposure, the RKN density in the soil treated with WiKim0069 (744 nematodes $/ 100 \mathrm{~g}$ ) was slightly lower than that in the soil treated with fosthiazate ( 886 nematodes $/ 100 \mathrm{~g}$ ).

\section{Discussion}

It has been widely demonstrated that lactobacilli exert an inhibitory effect on enteropathogens in human intestinal epithelial cells as well as on phytopathogenic bacteria and fungi. Recent studies have revealed that LAB can inhibit infection by phytopathogenic and spoilage bacteria and fungi, such as Xanthomonas campestris, Erwinia carotovora, Aspergillus flavus, Colletotrichum capsici, Penicillium expansum, Monilinia laxa, and Botrytis cinerea, and inhibit mycotoxin production (El-Mabrok et al., 2012; Ghazvini et al., 2016; Khanafari et al., 2007; Trias et al., 2008). Given the antagonistic activity of LAB against pathogenic microorganisms, the industrial application of LAB as a biocontrol microbe could be an effective strategy for phytopathogen management (Choi et al., 2018; Trias et al., 2008).

Lactobacillus plantarum has been considered as an antagonistic agent with antimicrobial activity, whereas little research has been performed on the potential of $L$. brevis as a biocontrol agent. Here, we evaluated the efficacy of LAB derived from the plant-based fermented food kimchi against RKNs. Of those tested, L. brevis WiKim0069 showed very strong in vitro mortality against $\mathrm{J} 2$ of $M$. incognita, $M$. arenaria, and M. hapla, and effectively suppressed gall formation on the roots of tomato and melon plants in pot and field experiments, respectively. We can suggest that L. brevis WiKim0069 is a novel bionematicide candidate and that $L$. brevis strains could be effective and eco-friendly alternatives to reduce the use of chemical nematicides in agriculture.

The artificial mixture of the four main organic acids produced by WiKim0069 caused 98\% J2 mortality of M. incognita at a $1.25 \%$ concentration, similar to that of the culture filtrate of $L$. brevis WiKim0069. These results indicate that the organic acids such as acetic acid, lactic acid, malic acid, and succinic acid are responsible for the nematicidal activity of the culture filtrate of $L$. brevis WiKim0069. Organic acids produced by LAB can decrease microbial populations by reducing the environment $\mathrm{pH}$ and subsequently reducing the internal $\mathrm{pH}$ of cells following the disruption of membrane permeability (Wódzki and Nowaczyk, 2001). The acidification of plant tissue can reduce the postharvest decay caused by Penicillium expansum and Alternaria alternate (Prusky et al., 2006). Furthermore, Choi et al. (2018) proposed that organic acids, especially lactic acid, may be more closely associated with the antagonistic activity of the LAB strains than other known antimicrobial compounds. However, of the four main organic acids produced by WiKim0069, acetic acid exhibited the strongest nematicidal activity against $M$. incognita $\mathrm{J} 2$ in vitro. Thus, our findings indicate that acetic acid may be a key nematicidal factor for RKN management, unlike the results of a previous study (Choi et al., 2018).

L. brevis WiKim0069 mainly produced acetic acid as the metabolic end product in MRS broth medium, followed by lactic acid, succinic acid, and malic acid. LAB generally produce lactic acid as the major metabolic end product of carbohydrate fermentation, whereas $L$. buchneri when added as an inoculant to whole maize crop silage was found 
to produce significantly more acetic acid than lactic acid (Driehuis et al., 1999). Although LAB are named after their ability to produce lactic acid, some lactobacilli, such as $L$. plantarum, L. brevis, L. buchneri, and L. bifermentans, are able to produce acetic acid from lactic acid under anoxic conditions at a pH $>4.0$ (da Cunha and Foster, 1992; Kandler et al., 1983; Lindgren et al., 1990). Here, the culture filtrate of L. brevis WiKim0069 had a pH of 4.2 and contained more acetic acid than lactic acid, indicating that $L$. brevis WiKim0069 may also degrade lactic acid to acetic acid.

Organic acids released during the decomposition of organic soil amendments have long been considered as alternative nematicides because they can decrease nematode populations (Akhtar and Malik, 2000; McBride et al., 2000). Additionally, studies on the nematicidal activity of microbial metabolites revealed that organic acids produced by several fungi exhibit strong nematicidal activity. Kojic acid produced by Aspergillus oryzae showed nematicidal activity against $M$. incognita (Kim et al., 2016). Among the $A$. niger culture metabolites, citric and oxalic acids were also demonstrated to have nematicidal activity (Jang et al., 2016; Zuckerman et al., 1994). Specifically, oxalic acid destroys internal bodies in $M$. incognita and produces numerous vacuoles in the nematode body. Acetic acid produced by the hyphomycetes Paecilomyces lilacinus and Trichoderma longibrachiatum was found to paralyze the J2 of M. incognita (Favre-Bonvin et al., 1991). Acetic acid also inhibits $\mathrm{J} 2$ hatching and affects embryogenesis (Bansal and Bajaj, 2003). In the case of bacteria, lactic acid isolated from Lysobacter capsici culture was also found to inhibit J2 hatching (Lee et al., 2014). However, the nematicidal activity of LAB against phytopathogenic nematodes has not been previously reported.

In bipolar ion-exchange membranes, the permeation of organic acids through the membrane appears to be competitive, and acetic acid among the carboxylic acids has a much higher permeability, followed by propionic, tartaric, lactic, oxalic, and citric acids (Wódzki and Nowaczyk, 2001). In M. incognita, exogenous treatment with acetic acid was found to damage the cuticle, degenerate the nuclei, and vacuolize the cytoplasm of nematodes (Ntalli et al., 2016). Application of L. brevis WiKim0069 also showed significant toxicity against $M$. incognita $\mathrm{J} 2$ and completely destroyed the internal organs by forming extensive vacuoles in the nematode body (Fig. 5). The target site of L. brevis WiKim0069 in the nematode body provided additional evidence that its nematicidal activity is mainly attributed to the organic acids it produces, including acetic acid. Based on the analysis of organic acids and their ne- maticidal activities, acetic acid was identified as the major nematicidal component of WiKim0069.

Seo and Kim (2014) reported that the mixture of acetic acid and lactic acid showed higher mortality against $\mathrm{J} 2$ of $M$. incognita than each organic acid of the two organic acids, and the nematicidal activity of the mixture was mostly derived from acetic acid. It has also been known that the nematicidal activity of organic acids highly depends on acidic $\mathrm{pH}$. However, we observed that the culture filtrates of LAB strains selected by primary screening showed different mortality each other and had $\mathrm{pH}$ in a range of 3.6 to 4.4 (Supplementary Table 2). Even though the culture filtrate of L. brevis WiKim0069 culture showed the strongest nematicidal activity among the test $237 \mathrm{LAB}$ strains in this study, its $\mathrm{pH}$ (4.2) was not the most acidic, but relatively less acidic among the $16 \mathrm{LAB}$ culture filtrates. Therefore, it is considered that there would be a great variation in the acid profiles from different bacteria and the antagonistic effect or synergy effect between the organic acids may affect their nematicidal activity. As a similar instance, the culture of different $\mathrm{LAB}$ strains with the same $\mathrm{pH}$ showed various inhibitory efficacy in vivo, suggesting that other factors involved in suppression of phytopathogens (Limanska et al., 2015).

WiKim0069 exhibited pronounced nematicidal activity against $M$. incognita compared with the untreated control. However, the inhibitory activity of L. brevis WiKim0069 was weaker than that of fosthiazate, a chemical nematicide, in pot experiments. Contrastingly, in melon field trials, the nematicidal activity of L. brevis WiKim0069 was higher than that of fosthiazate. It is difficult to clarify the mechanism of disease control under field conditions due to various synergistic interactions among soil microbes and their metabolites (Naseby et al., 2000). Several studies suggest that $\mathrm{LAB}$ can protect the host plants against soilborne diseases by root colonization and supply nutrients for plant healthy (Abdel-Aziz et al., 2014; Shrestha et al., 2014; Visser and Holzapfel, 1992). Treatment with various Lactobacillus strains can also improve the innate immunity of plants through a systemic acquired resistance, resulting in the upregulation of defense-related metabolites and leading to resistance to phytopathogens (Hamed et al., 2011; Konappa et al., 2016). Accordingly, the precise nematicidal mechanism of $L$. brevis WiKim0069 against RKNs has not yet been elucidated, but the high nematicidal efficacy of $L$. brevis WiKim0069 in the field trials provides the possibility that it may not only exhibit nematicidal activity but also interact with microorganisms in rhizospheric soil and/or activate plant defenses against RKNs.

Collectively, an RKN management strategy using LAB 
and their metabolites would meet the world's growing demand for eco-friendly nematicides that can replace synthetic chemicals and toxic pesticides in agriculture. However, prior to the commercial application of nematicidal LAB in agriculture, further studies on L. brevis WiKim0069 are needed to clarify the mechanism involved in the action of $\mathrm{LAB}$ against RKNs and develop the formulations of LAB for optimal performance under field conditions.

\section{Acknowledgements}

This study was supported by a grant from the World Institute of Kimchi (KE1901-1), funded by the Ministry of Science and ICT, Republic of Korea.

\section{Electronic Supplementary Material}

Supplementary materials are available at The Plant Pathology Journal website (http://www.ppjonline.org/).

\section{References}

Abbott, W. S. 1925. A method of computing the effectiveness of and insecticide. J. Econ. Entomol. 18:265-267.

Abdel-Aziz, S. M., Moustafa, Y. A. and Hamed, H. A. 2014. Lactic acid bacteria in the green biocontrol against some phytopathogenic fungi: treatment of tomato seeds. J. Basic Appl. Sci. Res. 4:1-9.

Akhtar, M. and Malik, A. 2000. Roles of organic soil amendments and soil organisms in the biological control of plantparasitic nematodes: a review. Bioresour. Technol. 74:35-47.

Aktar, M. W., Sengupta, D. and Chowdhury, A. 2009. Impact of pesticides use in agriculture: their benefits and hazards. Interdiscip. Toxicol. 2:1-12.

Ashoub, A. H. and Amara, M. T. 2010. Biocontrol activity of some bacterial genera against root-knot nematode, Meloidogyne incognita. J. Am. Sci. 6:321-328.

Bansal, R. K. and Bajaj, A. 2003. Effect of volatile fatty acids on embryogenesis and hatching of Meloidogyne incognita eggs. Nematol. Mediterr. 31:135-140.

Barefoot, S. F. and Klaenhammer, T. R. 1983. Detection and activity of lactacin B, a bacteriocin produced by Lactobacillus acidophilus. Appl. Environ. Microbiol. 45:1808-1815.

Barker, K. R. 1985. Nematode extractions and bioassays. In: An advanced treatise on Meloidogyne, Vol. II. Methodology, eds. by K. R. Barker, C. C. Carter, J. N. Sasser, pp. 19-35. North Carolina State University Graphics, Raleigh, NC, USA.

Blok, V. C., Jones, J. T., Phillips, M. S. and Trudgill, D. L. 2008. Parasitism genes and host range disparities in biotrophic nematodes: the conundrum of polyphagy versus specialisation. BioEssays 30:249-259.

Cayrol, J.-C., Djian, C. and Pijarowski, L. 1989. Study of the ne- matocidal properties of the culture filtrate of the nematophagous fungus Paecilomyces lilacinus. Rev. Nematol. 12:331336.

Choi, A.-R., Patra, J. K., Kim, W. J. and Kang, S.-S. 2018. Antagonistic activities and probiotic potential of lactic acid bacteria derived from a plant-based fermented food. Front. Microbiol. 9:1963.

da Cunha, M. V. and Foster, M. A. 1992. Sugar-glycerol cofermentations in lactobacilli: the fate of lactate. J. Bacteriol. 174:1013-1019.

Driehuis, F., Elferink, S. J. and Spoelstra, S. F. 1999. Anaerobic lactic acid degradation during ensilage of whole crop maize inoculated with Lactobacillus buchneri inhibits yeast growth and improves aerobic stability. J. Appl. Microbiol. 87:583594.

El-Mabrok, A. S. W., Hassan, Z., Mokhtar, A. M., Hussain, K. M. A. and Kahar, F. K. S. B. A. 2012. Screening of lactic acid bacteria as biocontrol against (Collectotrichum capsici) on chilli Bangi. Res. J. Appl. Sci. 7:446-473.

Elling, A. A. 2013. Major emerging problems with minor Meloidogyne species. Phytopathology 103:1092-1102.

Favre-Bonvin, J., Ponchet, M., Djian, C., Arpin, N. and Pijarowski, L. 1991. Acetic acid: a selective nematicidal metabolite from culture filtrates of Paecilomyces lilacinus (Thom) Samson and Trichoderma longibrachiatum Rifai. Nematologica 37:101-112.

Ghazvini, R. D., Kouhsari, E., Zibafar, E., Hashemi, S. J., Amini, A. and Niknejad, F. 2016. Antifungal activity and aflatoxin degradation of Bifidobacterium bifidum and Lactobacillus fermentum against toxigenic Aspergillus parasiticus. Open Microbiol. J. 10:197-201.

Gilliland, S. E. 1990. Health and nutritional benefits from lactic acid bacteria. FEMS Microbiol. Rev. 7:175-188.

Guarner, F., Khan, A. G., Garisch, J., Eliakim, R., Gangl, A., Thomson, A., Krabshuis, J., Lemair, T., Kaufmann, P., de Paula, J. A., Fedorak, R., Shanahan, F., Sanders, M. E., Szajewska, H., Ramakrishna, B. S., Karakan, T., Kim, N. and World Gastroenterology Organization. 2012. World Gastroenterology Organisation global guidelines: probiotics and prebiotics October 2011. J. Clin. Gastroenterol. 46:468-481.

Hamed, H. A., Moustafa, Y. A. and Abdel-Aziz, S. M. 2011. In vivo efficacy of lactic acid bacteria in biological control against Fusarium oxysporum for protection of tomato plant. Life Sci. J. 8:462-468.

Holbrook, C. C., Knauft, D. A. and Dickson, D. W. 1983. A technique for screening peanut for resistance to Meloidogyne arenaria. Plant Dis. 67:957-958.

Hwang, S. M., Park, M. S., Kim, J.-C., Jang, K. S., Choi, Y. H. and Choi, G. J. 2014. Occurrence of Meloidogyne incognita infecting resistant cultivars and development of an efficient screening method for resistant tomato to the $M i$-virulent nematode. Korean J. Hortic. Sci. Technol. 32:217-226.

Jang, J. Y., Choi, Y. H., Shin, T. S., Kim, T. H., Shin, K.-S., Park, H. W., Kim, Y. H., Kim, H., Choi, G. J., Jang, K. S., Cha, 
B., Kim, I. S., Myung, E. J. and Kim, J.-C. 2016. Biological control of Meloidogyne incognita by Aspergillus niger F22 producing oxalic acid. PLOS ONE 11:e0156230.

Jang, S.-E., Hyun, Y.-J., Oh, Y.-J., Choi, K. B., Kim, T., Yeo, I. H., Han, M. J. and Kim, D.-H. 2011. Adhesion activity of Lactobacillus plantarum PM 008 isolated from kimchi on the intestine of mice. J. Bacteriol. Virol. 41:83-90.

Jenkins, W. R. 1964. A rapid centrifugal-flotation technique for separating nematodes from soil. Plant Dis. Rep. 48:692.

Jones, J. T., Haegeman, A., Danchin, E. G. J., Gaur, H. S., Helder, J., Jones, M. G. K., Kikuchi, T., Manzanilla-López, R., Palomares-Rius, J. E., Wesemael, W. M. L. and Perry, R. N. 2013. Top 10 plant-parasitic nematodes in molecular plant pathology. Mol. Plant Pathol. 14:946-961.

Jung, J. Y., Lee, S. H., Kim, J. M., Park, M. S., Bae, J.-W., Hahn, Y., Madsen, E. L. and Jeon, C. O. 2011. Metagenomic analysis of kimchi, a traditional Korean fermented food. Appl. Environ. Microbiol. 77:2264-2274.

Kandler, O., Schillinger, U. and Weiss, N. 1983. Lactobacillus bifermentans sp. nov., nom. rev., an organism forming $\mathrm{CO}_{2}$ and $\mathrm{H}_{2}$ from lactic acid. Syst. Appl. Microbiol. 4:408-412.

Khanafari, A., Soudi, H. and Miraboulfathi, M. 2007. Biocontrol of Aspergillus flavus and aflatoxin B1 production in corn. Iran. J. Environ. Health Sci. Eng. 4:163-168.

Kim, D.-G., Lee, Y.-K. and Park, B.-Y. 2001. Root-knot nematode species distributing in greenhouses and their simple identification scheme. Res. Plant Dis. 7:49-55 (in Korean).

Kim, T. Y., Jang, J. Y., Jeon, S. J., Lee, H. W., Bae, C.-H., Yeo, J. H., Lee, H. B., Kim, I. S., Park, H. W. and Kim, J.-C. 2016. Nematicidal activity of kojic acid produced by Aspergillus oryzae against Meloidogyne incognita. J. Microbiol. Biotechnol 26:1383-1391.

Konappa, N. M., Maria, M., Uzma, F., Krishnamurthy, S., Nayaka, S. C., Niranjana, S. R. and Chowdappa, S. 2016. Lactic acid bacteria mediated induction of defense enzymes to enhance the resistance in tomato against Ralstonia solanacearum causing bacterial wilt. Sci. Hortic. 207:183-192.

Kormin, S., Rusul, G., Radu, S. and Ling, F. H. 2001. Bacteriocin-producing lactic acid bacteria isolated from traditional fermented food. Malays. J. Med. Sci. 8:63-68.

Lee, Y. S., Naning, K. W., Nguyen, X. H., Kim, S. B., Moon, J. H. and Kim, K. Y. 2014. Ovicidal activity of lactic acid produced by Lysobacter capsici YS1215 on eggs of root-knot nematode, Meloidogyne incognita. J. Microbiol. Biotechnol. 24:1510-1515.

Lim, J.-H., Yoon, S.-M., Tan, P.-L., Yang, S., Kim, S.-H. and Park, H.-J. 2018. Probiotic properties of Lactobacillus Plantarum LRCC5193, a plant-origin lactic acid bacterium isolated from kimchi and its use in chocolates. J. Food Sci. 83:2802-2811.

Limanska, N., Korotaeva, N., Biscola, V., Ivanytsia, T., Merlich, A., Franco, B. D. G. M. and Haertlé, T. 2015. Study of the potential application of lactic acid bacteria in the control of infection caused by Agrobacterium tumefaciens. J. Plant
Pathol. Microbiol. 6:292.

Lindgren, S. E., Axelsson, L. T. and McFeeters, R. F. 1990. Anaerobic L-lactate degradation by Lactobacillus plantarum. FEMS Microbiol. Lett. 66:209-213.

Lindgren, S. E. and Dobrogosz, W. J. 1990. Antagonistic activities of lactic acid bacteria in food and feed fermentations. FEMS Microbiol. Rev. 7:149-163.

McBride, R. G., Mikkelsen, R. L. and Barker, K. R. 2000. The role of low molecular weight organic acids from decomposing rye in inhibiting root-knot nematode populations in soil. Appl. Soil Ecol. 15:243-251.

Meyer, S. L. F., Massoud, S. I., Chitwood, D. J. and Roberts, D. P. 2000. Evaluation of Trichoderma virens and Burkholderia cepacia for antagonistic activity against root-knot nematode, Meloidogyne incognita. Nematology 2:871-879.

Moens, M., Perry, R. N. and Starr, J. L. 2009. Meloidogyne species: a diverse group of novel and important plant parasites. In: Root-knot nematodes, eds. by R. N. Perry, J. L. Starr and M. Moens, pp. 1-17. CAB International, London, UK.

Molinari, S. 2009. Antioxidant enzymes in (a)virulent populations of root-knot nematodes. Nematology 11:689-697.

Molinari, S., Fanelli, E. and Leonetti, P. 2014. Expression of tomato salicylic acid (SA)-responsive pathogenesis-related genes in $\mathrm{Mi}$-1-mediated and SA-induced resistance to rootknot nematodes. Mol. Plant Pathol. 15:255-264.

Naseby, D. C., Pascual, J. A. and Lynch, J. M. 2000. Effect of biocontrol strains of Trichoderma on plant growth, Pythium ultimum populations, soil microbial communities and soil enzyme activities. J. Appl. Microbiol. 88:161-169.

Ntalli, N., Ratajczak, M., Oplos, C., Menkissoglu-Spiroudi, U. and Adamski, Z. 2016. Acetic acid, 2-undecanone, and (e)2-decenal ultrastructural malformations on Meloidogyne incognita. J. Nematol. 48:248-260.

Park, J., Seo, Y. and Kim, Y. H. 2014. Biological control of Meloidogyne hapla using an antagonistic bacterium. Plant Pathol. J. 30:288-298.

Prusky, D., Kobiler, I., Akerman, M. and Miyara, I. 2006. Effect of acidic solutions and acidic prochloraz on the control of postharvest decay caused by Alternaria alternata in mango and persimmon fruit. Postharvest Biol. Technol. 42:134-141.

Ralmi, N. H. A. A., Khandaker, M. M. and Mat, N. 2016. Occurrence and control of root knot nematode in crops: a review. Aust. J. Crop Sci. 10:1649-1654.

Schneider, P. and Orelli, O. 1947. Entomologisches praktikum [Entomological internship]. Verlag. H. R. Sauerländer Co., Aarau, Switzerland. 237 pp.

Seo, Y. and Kim, Y. H. 2014. Control of Meloidogyne incognita using mixtures of organic acids. Plant Pathol. J. 30:450-455.

Shrestha, A., Kim, B. S. and Park, D. H. 2014. Biological control of bacterial spot disease and plant growth-promoting effects of lactic acid bacteria on pepper. Biocontrol Sci. Technol. 24:763-779.

Sitton, J. W. and Patterson, M. E. 1992. Effect of high-carbon dioxide and low-oxygen controlled atmospheres on postharvest 
decays of apples. Plant Dis. 76:992-995.

Stiles, M. E. and Holzapfel, W. H. 1997. Lactic acid bacteria of foods and their current taxonomy. Int. J. Food Microbiol. 36:1-29.

Tamura, K., Stecher, G., Peterson, D., Filipski, A. and Kumar, S. 2013. MEGA6: molecular evolutionary genetics analysis version 6.0. Mol. Biol. Evol. 30:2725-2729.

Terefe, M., Tefera, T. and Sakhuja, P. K. 2009. Effect of a formulation of Bacillus firmus on root-knot nematode Meloidogyne incognita infestation and the growth of tomato plants in the greenhouse and nursery. J. Invertebr. Pathol. 100:94-99.

Trias, R., Bañeras, L., Montesinos, E. and Badosa, E. 2008. Lactic acid bacteria from fresh fruit and vegetables as biocontrol agents of phytopathogenic bacteria and fungi. Int. Microbiol. 11:231-236.

Trudgill, D. L. and Blok, V. C. 2001. Apomictic, polyphagous root-knot nematodes: exceptionally successful and damaging biotrophic root pathogens. Annu. Rev. Phytopathol 39:53-77.

Viglierchio, D. R. and Schmitt, R. V. 1983. On the methodology of nematode extraction from field samples: Baermann funnel modifications. J. Nematol. 15:438-444.

Visser, R. and Holzapfel, W. H. 1992. Lactic acid bacteria in the control of plant pathogens. In: The Lactic Acid Bacteria. Vol. 1, ed. by B. J. B. Wood, pp. 193-210. Springer, Boston, MA, USA.

Wódzki, R. and Nowaczyk, J. 2001. Membrane transport of organics. III. Permeation of some carboxylic acids through bipolar polymer membrane. J. Appl. Polym. Sci. 80:2705-2717.

Yadav, U. 2017. Recent trends in nematode management practices: the Indian context. Int. Res. J. Eng. Technol. 4:482-489.

Yeon, J., Park, A. R., Kim, Y. J., Seo, H. J., Yu, N. H., Ha, S., Park, H. W. and Kim, J.-C. 2019. Control of root-knot nematodes by a mixture of maleic acid and copper sulfate. Appl. Soil Ecol. 141:61-68.

Zuckerman, B. M., Matheny, M. and Acosta, N. 1994. Control of plant-parasitic nematodes by a nematicidal strain of Aspergillus niger. J. Chem. Ecol. 20:33-43. 